\title{
On the strong Hanani-Tutte Theorem
}

\author{
Hooman R. Dehkordi Graham Farr \\ Faculty of Information Technology \\ Monash University \\ Clayton, Victoria 3800, Australia \\ \{hooman.dehkordi, graham.farr\}@monash.edu
}

Submitted: Jul 28, 2019; Accepted: Feb 2, 2021; Published: Feb 26, 2021

(C) The authors. Released under the CC BY license (International 4.0).

\begin{abstract}
A graph is planar if it has a drawing in which no two edges cross. The HananiTutte Theorem states that a graph is planar if it has a drawing $D$ such that any two edges in $D$ cross an even number of times.

A graph $G$ is a non-separating planar graph if it has a drawing $D$ such that (1) edges do not cross in $D$, and (2) for any cycle $C$ and any two vertices $u$ and $v$ that are not in $C, u$ and $v$ are on the same side of $C$ in $D$. Non-separating planar graphs are closed under taking minors and hence have a finite forbidden minor characterisation.

In this paper, we prove a Hanani-Tutte type theorem for non-separating planar graphs. We use this theorem to prove a stronger version of the strong Hanani-Tutte Theorem for planar graphs, namely that a graph is planar if it has a drawing in which any two disjoint edges cross an even number of times or it has a chordless cycle that enables a suitable decomposition of the graph.
\end{abstract}

Mathematics Subject Classifications: 05C10, $05 \mathrm{C} 83$

\section{Introduction}

The Hanani-Tutte Theorem is a well-known theorem about planar graphs:

Strong Hanani-Tutte Theorem ([12]). A graph is planar if it has a drawing $D$ on the plane such that any two vertex-disjoint edges in $D$ cross each other an even number of times.

A drawing $D$ of a graph $G$ in a plane $\Sigma$ partitions all the points of $\Sigma \backslash D$ (where here by $D$ we also denote the set of all points in $D$ ) into a set of regions, denoted by regions $(D)$, such that any two points $p$ and $q$ are in the same region $r \in$ regions $(D)$ if and only if there is a curve from $p$ to $q$ that does not cross any vertex or edge of $D$. Two regions are adjacent if they share an edge or a segment of an edge on their boundaries. 
For any drawing $D$ of a cycle, define a black-and-white colouring of the plane with respect to $D$ to be a colouring of each region of regions $(D)$ either black or white such that no two adjacent regions are coloured in the same colour (see, for example, Figure 1). Existance of such 2-colouring is well-known, however to make the paper self-contained we give a short proof for this in Lemma 12.

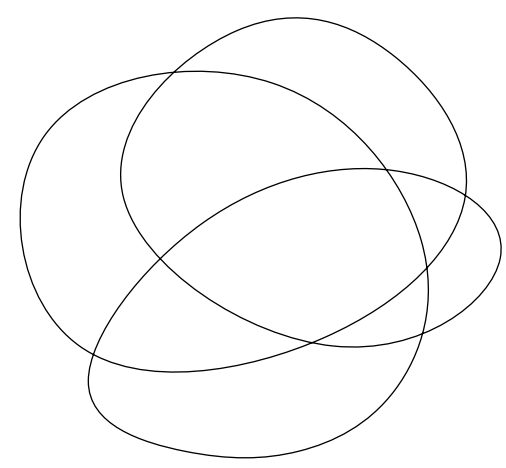

(a) A drawing $D$ of cycle $C$

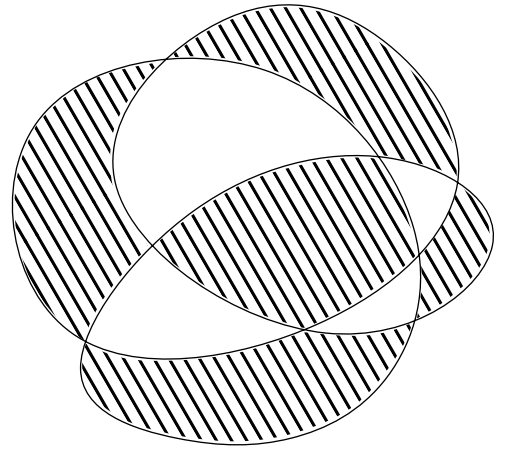

(b) Colouring regions of the plane in black and white based on $D$

Figure 1

Let $D$ be a drawing of a graph $G$ and let $G^{-}=G[S]$, where $S \subseteq V(G)$. Then $D\left[G^{-}\right]$ is a drawing of $G^{-}$that is obtained as follows:

- for each vertex $v$ in $G^{-}$, let $v$ be represented by the same point that represents $v$ in $D$, and

- for each edge $e$ in $G^{-}$, let $e$ be represented by the same curve that represents $e$ in $D$.

Let $C$ be a cycle in a graph $G$. Let $D$ be a drawing of $G$. Cycle $C$ is a separating cycle in $D$ if there is a pair of vertices $u$ and $v$ in $G$ such that:

- $u \notin V(C), v \notin V(C)$ and

- $u$ and $v$ are located in regions with different colours in a black-white colouring of the plane with respect to $D[C]$.

We say that cycle $C$ separates $u$ from $v$ in $D$.

A non-separating drawing of a graph is a drawing of the graph that does not contain any separating cycles. A non-separating planar graph is a graph that has a non-separating planar drawing.

Non-separating planar graphs are a proper subclass of planar graphs and a proper superclass of outerplanar graphs. They are closed under minors. The graph $K_{4}$ is an example of a non-separating planar graph that is not outerplanar.

In this paper we first prove a Hanani-Tutte type theorem for non-separating planar graphs. 
Theorem 1. Let $D$ be a non-separating drawing of a graph $G$ such that any two vertexdisjoint edges in $D$ cross each other an even number of times. Then $G$ is a non-separating planar graph.

We then use the Theorem 1 to prove a stronger version of the Hanani-Tutte Theorem. To state the stronger version of the Hanani-Tutte Theorem, first we need to define a couple of new terms.

A separating cycle $C$ in $D$ is a decomposing cycle if any edge $e$ that is vertex-disjoint from $C$ crosses $C$ an even number of times in $D$. For a black-and-white colouring of regions of the plane with respect to $D[C]$, where $C$ is a decomposing cycle, let $V_{w}$ be the set of vertices of $G$ that are on a white region of the plane and let $V_{b}$ be the set of vertices of $G$ that are on a black region of the plane. Let $G_{w}$ be the graph that is induced from $G$ by the vertices of $C$ and $V_{w}\left(G_{w}=G\left[V_{w} \cup V(C)\right]\right)$ and let $G_{b}$ be the graph that is induced from $G$ by the vertices of $C$ and $V_{b}\left(G_{b}=G\left[V_{b} \cup V(C)\right]\right)$. Note that $C$ is a part of both $G_{w}$ and $G_{b}$. Then we say that $C$ decomposes $D$ into two drawings: $D\left[G_{b}\right]$ and $D\left[G_{w}\right]$.

A drawing $D$ is evenly decomposable if:

- every two vertex-disjoint edges in $D$ cross an even number of times, or

- there is a chordless decomposing cycle that decomposes $D$ into two evenly decomposable drawings.

A planar drawing is a drawing in which no two edges cross.

Now we can state a stronger version of the Hanani-Tutte Theorem.

Theorem 2. Let $D$ be an evenly decomposable drawing of a graph $G$ on the plane. Then $G$ is planar and there is a planar drawing $D^{\prime}$ of $G$ such that any decomposing chordless cycle $C$ in $S$ separates vertices $u$ and $v$ in $D^{\prime}$ if and only if $C$ separates $u$ and $v$ in $D$.

Since a drawing in which any two vertex-disjoint edges cross each other an even number of times is evenly decomposable, Theorem 2 is at least as strong as the strong version of the Hanani-Tutte Theorem. Moreover since there are evenly decomposable drawings in which there is a pair of vertex-disjoint edges that cross each other an odd number of times, Theorem 2 is stronger than the strong version of the Hanani-Tutte Theorem.

Figure 2 depicts a simple example of an evenly decomposable drawing with a pair of vertex-disjoint edges that cross an odd number of times.

The rest of this paper is organised as follows. Section 2 reviews previous work and puts the results of this paper into the context. Section 3 is dedicated to proving a number of preliminary lemmas. Section 4 is dedicated to proving Theorem 1 . Theorem 1 will later be used in the proof of Theorem 2. In Section 5 we prove Theorem 2. Lastly, in Section 6, we summarise our results and suggest some future directions for research.

\section{Background}

A drawing of a graph in the plane consists of a set of points representing (under a bijection) the vertices of the graph and a set of curves between certain pairs of points representing 

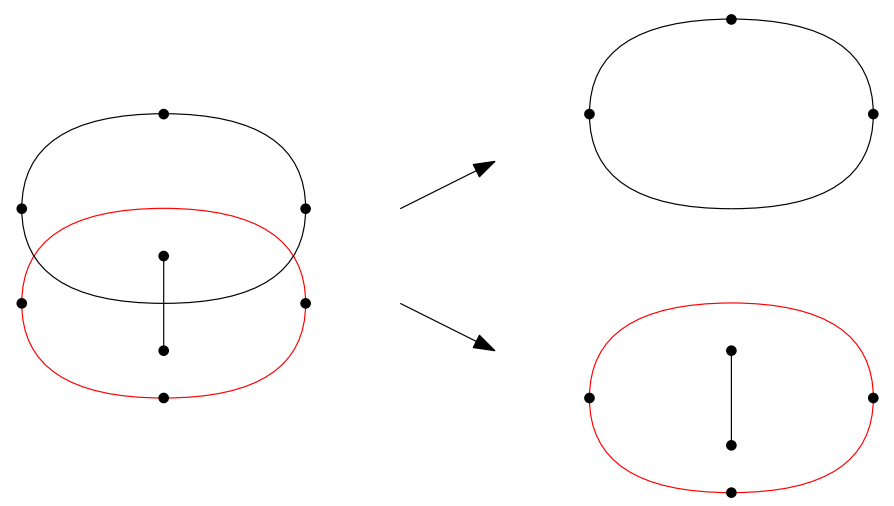

Figure 2: An evenly decomposable drawing with two edges that cross each other an odd number of times. The cycle drawn in red is the decomposing cycle in this drawing.

edges between corresponding pairs of vertices of the graph. Two edges cross if they share a point other than their endpoints. The shared point is a crossing.

In this paper we assume that a drawing $D$ satisfies the following conditions:

- An edge does not contain a vertex other than its endpoints.

- Edges must not meet tangentially, that is, they must either properly cross or not cross at all. So crossings must be transversal intersections.

- No point represents two crossings.

We sometimes also use $D$ to denote the set of all points in $D$.

A planar drawing is a drawing in which no two edges cross. Planar graphs are the graphs that have a planar drawing.

An edge $e=(u, v)$ in a graph $G$ is subdivided by replacing it with two edges $(u, w)$, $(w, v)$ where $w$ is not a vertex of $G$. A subdivision of a graph $G$ is a graph that can be obtained from $G$ by a sequence of subdivisions (or no subdivisions). Kuratowski's well-known theorem characterises planar graphs in terms of two forbidden subdivisions.

Kuratowski's Theorem (Kuratowki [8]). A graph is planar if and only if it does not contain a subdivision of $K_{5}$ or a subdivision of $K_{3,3}$ as a subgraph.

The Hanani-Tutte Theorem is another famous result in graph theory which can be thought of as a characterisation of planar graphs in terms of their drawings. Hanani proved the following in 1934.

Theorem 3 (Hanani [3]). Every drawing of $K_{5}$ or $K_{3,3}$ contains two vertex-disjoint edges that cross an odd number of times.

By Kuratowski's Theorem and Hanani's Theorem, we can easily see that in any drawing of a non-planar graph there are two vertex-disjoint paths that cross an odd number of times and hence there are two vertex-disjoint edges that cross an odd number of times. In other words, it is easy to derive the strong Hanani-Tutte Theorem from Theorem 3. 
The strong Hanani-Tutte Theorem was first explicitly stated by Tutte [12]. This Theorem is usually used in the following weaker form known as the weak Hanani-Tutte Theorem:

Theorem 4 (weak Hanani-Tutte Theorem). A graph is planar if it has a drawing D such that any two edges in $D$ cross an even number of times.

The difference between the strong version and the weak version of the Hanani-Tutte Theorem is that in the strong Hanani-Tutte we only require the vertex-disjoint pairs of edges in a drawing of the graph to cross each other an even number of times whereas in the weak Hanani-Tutte Theorem we require any pair of edges in a drawing of a graph to cross an even number of times.

Notice that both the strong and the weak Hanani-Tutte Theorems provide us with a characterisation of planar graphs since the converse of both of these theorems is true. More specifically, by definition any planar graph has a drawing in which any two edges cross zero times.

The Hanani-Tutte Theorem in its weak form has been generalised to all orientable surfaces in 2000 [2] and to all 2-manifolds in 2005 [11]. This is especially interesting as we do not yet have forbidden minor characterisations for the graphs that can be drawn without crossings on surfaces other than the sphere and projective plane.

Theorem 5 ([11]). For every surface $\Sigma$, a graph $G$ has a drawing on $\Sigma$ with no crossings if and only if it has a drawing $D$ on $\Sigma$ such that any two edges cross an even number of times in $D$.

However, the strong Hanani-Tutte Theorem has only been generalised for the projective plane [10]. In fact we know that it can not be generalised for the surface of genus four [5].

Theorem 6 ([10]). A graph $G$ has a drawing on the projective plane with no crossings if and only if it has a drawing $D$ on the projective plane such that any two vertex-disjoint edges cross an even number of times in $D$.

Theorem 7 ([5]). There exists a graph $G$ with a drawing in the compact orientable surfaces $\Sigma$ with 4 handles in which every pair of vertex-disjoint edges cross an even number of times such that $G$ does not have a planar drawing in $\Sigma$.

There are numerous other versions of the Hanani-Tutte Theorem. For example, see the following slightly stronger version of the weak Hanani-Tutte Theorem:

Theorem 8 ([9, 11]). Let $D$ be a drawing of a graph $G$ such that any two edges cross an even number of times in $D$. Then $G$ is planar and has a planar drawing $D^{\prime}$ with a rotation system of edges around the vertices that is the same as the rotation system of edges around the vertices in $D$. 


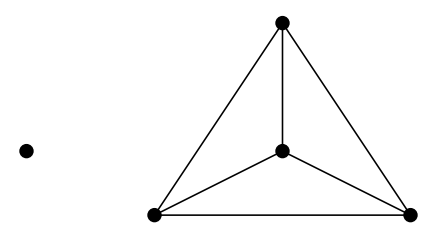

(a) $K_{1} \cup K_{4}$

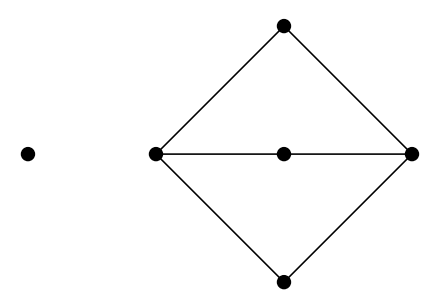

(b) $K_{1} \cup K_{2,3}$

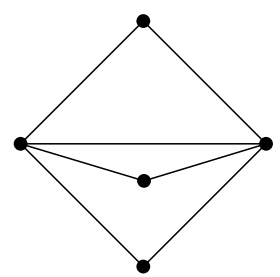

(c) $K_{1,1,3}$

Figure 3: Excluded minors for non-separating planar graphs

The conclusion of Theorem 8 is often assumed to be included in the weak Hanani-Tutte Theorem.

Recently, Fulek, Kynčl and Pálvölgyi unified this version of the weak Hanani-Tutte Theorem with the Strong Hanani-Tutte Theorem as follows:

Theorem 9 ([6]). Let $G$ be a graph and let $W \subseteq V(G)$. Let $D$ be a drawing of $G$ where every pair of edges that are vertex-disjoint or have a common endpoint in $W$ cross an even number of times. Then $G$ has a planar drawing where the cyclic orders of edges at vertices of $W$ are the same as in $D$.

If $W$ is empty, in the above theorem, then the theorem is equivalent to the strong version of the Hanani-Tutte Theorem and if $W=V(G)$ then the theorem is equivalent to the weak version of the Hanani-Tutte Theorem.

Non-separating planar graphs are a subclass of planar graphs and a superclass of outerplanar graphs. They are closed under minors. Dehkordi and Farr gave a forbidden minor characterisation of this class of graphs as follows:

Theorem 10 ([4]). A graph $G$ is a non-separating planar graph if and only if it does not contain any of $K_{1} \cup K_{4}$ or $K_{1} \cup K_{2,3}$ or $K_{1,1,3}$ as a minor.

A graph is a triangular prism if it is isomorphic to the graph that is depicted in Figure 4. A graph is an elongated triangular prism if it is a triangular prism or if it is obtained by some sequence of subdivisions of some or all of the red dashed edges of the triangular prism depicted in Figure 4.

Dehkordi and Farr have also proved the following structural characterisation of nonseparating planar graphs:

Theorem 11 ([4]). Every non-separating planar graph is one of the following:

1. an outerplanar graph,

2. a subgraph of a wheel,

3. a subgraph of an elongated triangular prism.

In this paper we prove Theorem 1 which is a Hanani-Tutte type theorem for nonseparating planar graphs.

We then use Theorem 1 to prove a stronger version of the Hanani-Tutte Theorem, namely Theorem 2 . 


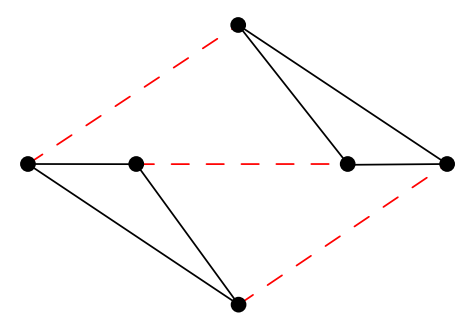

Figure 4: A triangular prism where some of the edges are depicted as dashed edges.

\section{Preliminary Results}

Lemma 12. Let $D$ be a drawing of a cycle on the plane. Then there is a black-and-white colouring for $D$.

Proof. Let $G$ be a graph with a vertex for each region of $D$ and an edge $(u, v)$ for any two adjacent regions $r_{1}$ and $r_{2}$ of $D$ where $r_{1}$ is represented by vertex $u$ of $G$ and $r_{2}$ is represented by vertex $v$ of $G$.

Graph $G$ is a dual of an Eulerian planar graph and therefore it is bipartite.

A realisation of a graph is a drawing of it in three-dimensional space $\mathbb{R}^{3}$ (see for example Figure 5a). A projection of a graph $G$ is a drawing of $G$ with an over/under relation between the edges specified at each crossing (see for example Figure 5b).

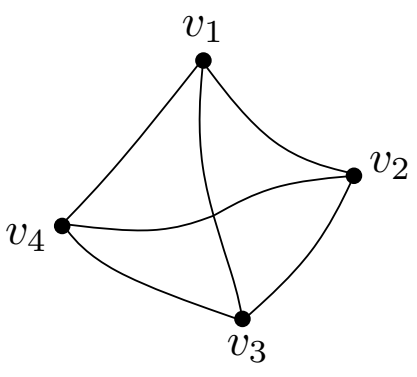

(a) A drawing of $K_{4}$

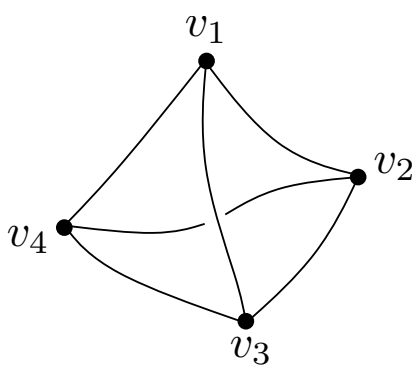

(b) A projection of $K_{4}$

Figure 5: Difference between a drawing and a projection of $K_{4}$

Kaufmann [7] and Yamada [13] independently proved that if two (piecewise linear) projections $\mathcal{P}$ and $\mathcal{P}^{\prime}$ represent the same embedding of a graph in $\mathbb{R}^{3}$ then $\mathcal{P}$ and $\mathcal{P}^{\prime}$ are related by a finite sequence of the local moves as stated in the following theorem.

Theorem 13 (Kaufmann [7] and Yamada [13]). Let $\mathcal{P}$ and $\mathcal{P}^{\prime}$ be two projections of the same realisation of a graph. Then one can obtain $\mathcal{P}^{\prime}$ from $\mathcal{P}$ by a finite sequence of the local moves given in Table 1.

Using Theorem 13 it is easy to prove that one can change any drawing of a graph $G$ in the plane to any other drawing of $G$ in the plane using the moves in Table 2. 


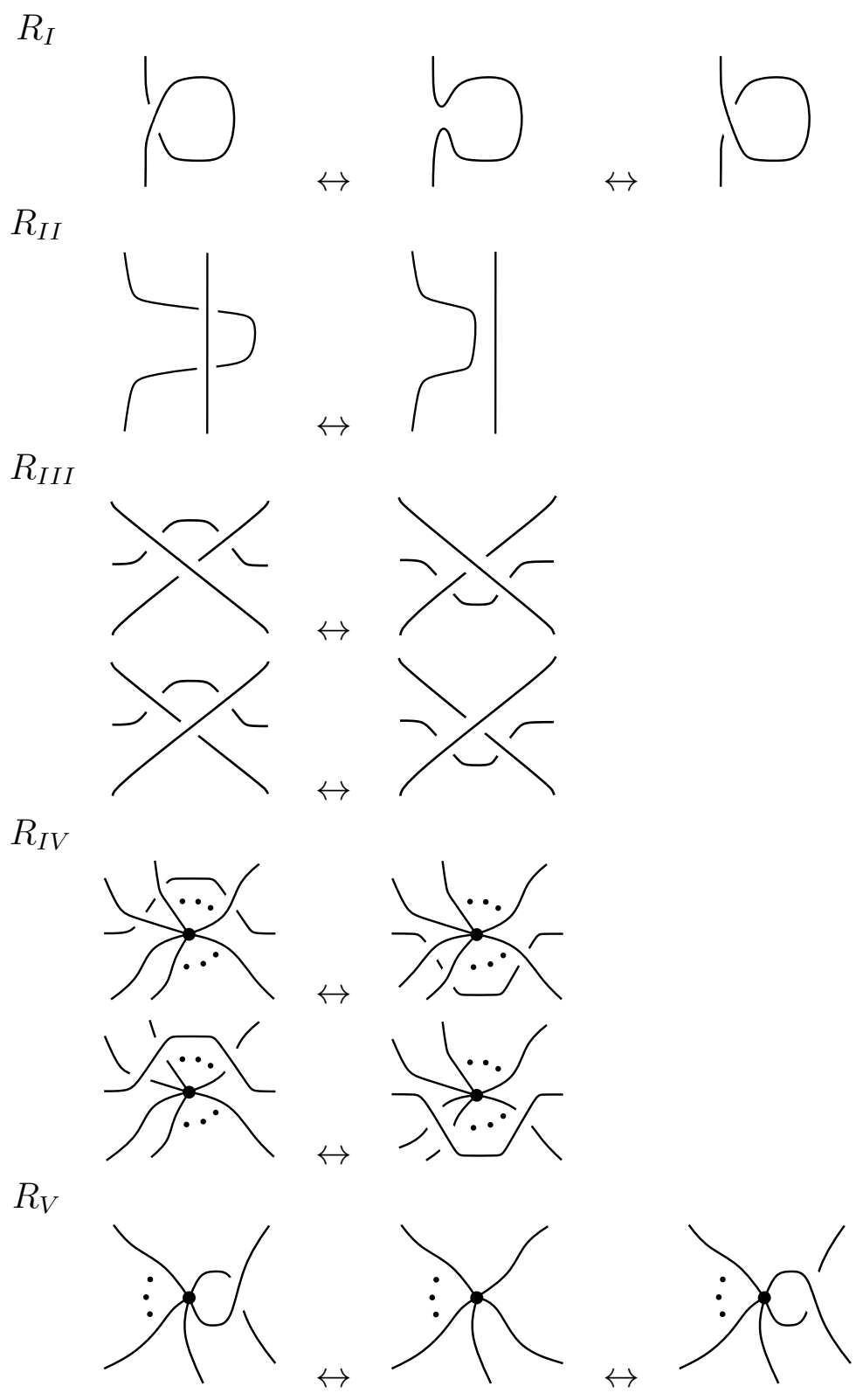

Table 1: Reidemeister moves for spatial graphs 
Proposition 14. Let $D$ and $D^{\prime}$ be two drawings of a graph $G$ in the plane. Then $D$ and $D^{\prime}$ are related by a finite sequence of the local moves given in Table 2.

Proof. Convert $D$ and $D^{\prime}$ to two projections $\mathcal{P}$ and $\mathcal{P}^{\prime}$ of $G$ by changing the crossings in $D$ and $D^{\prime}$ to overcrossings and undercrossings arbitrarily. $\mathcal{P}$ and $\mathcal{P}^{\prime}$ are projections of two spatial embeddings of $G$, which may not be ambient isotopic. However, any spatial embedding of a graph $G$ can be converted to any other spatial embedding of $G$ if we allow the edges to deform (ambient isotopy) and pass through each other (see, for example, [1], page 219). That is, by Theorem 13 we can change $\mathcal{P}$ to $\mathcal{P}^{\prime}$ by a finite sequence of Reidemeister moves from the set given in Table 1 and changing some overcrossings to undercrossings and some undercrossings to overcrossings.

Obtain $\mathcal{P}^{\prime}$ from $\mathcal{P}$ using a finite number $k$ of steps, where each step is either a single Reidemeister move from Table 1 or changing an overcrossing to an undercrossing or vice versa. Then change the undercrossings and overcrossings to simple crossings in order to obtain $D^{\prime}$ from $\mathcal{P}^{\prime}$. Let $\mathcal{P}_{0}=\mathcal{P}$ and let $\mathcal{P}_{i}$ represent the projection that is obtained by applying the first $i$ steps to $\mathcal{P}$ and let $D_{i}$ be the drawing of $G$ in the plane that is obtained by changing overcrossings and undercrossings of $\mathcal{P}_{i}$ to crossings (see Figure 6 ).

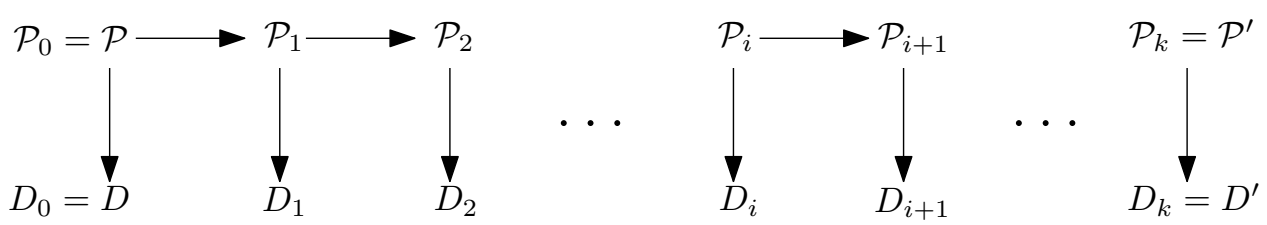

Figure 6: Transforming $\mathcal{P}$ to $\mathcal{P}^{\prime}$ through a series of steps using Reidemeister moves from Table 1 and changes between overcrossings and undercrossings. Any horizontal arrow in this picture represents either a Reidemeister move or a change of an undercrossing to overcrossing or vice versa. Any downward vertical arrow represents a transformation of a projection to a drawing by changing overcrossings or undercrossings to crossings.

The transformation of $\mathcal{P}$ to $\mathcal{P}^{\prime}$ via the intermediate steps $\mathcal{P}_{i} \rightarrow \mathcal{P}_{i+1}$, together with the crossing removals that reduce $\mathcal{P}_{i}$ to $D_{i}$, yield a transformation of $D$ to $D^{\prime}$ through a series of steps by transforming each $D_{i}$ to $D_{i+1}$. In each step we can obtain $D_{i+1}$ from $D_{i}$, for all $i \in 0, \ldots, k-1$, by:

1. Obtaining the projection $\mathcal{P}_{i}$ from $D_{i}$ by changing the crossings of $D_{i}$ to overcrossings and undercrossings according to $\mathcal{P}_{i}$.

2. Using one of the Reidemeister moves in Table 1, or changing an undercrossing to an overcrossing or vice versa, to change $\mathcal{P}_{i}$ to $\mathcal{P}_{i+1}$.

3. Obtaining a drawing $D_{i+1}$ from $\mathcal{P}_{i+1}$ by changing overcrossings and undercrossings of $\mathcal{P}_{i+1}$ to crossings.

If the transformation of $\mathcal{P}_{i}$ to $\mathcal{P}_{i+1}$ is obtained by a change of an overcrossing to an undercrossing or vice versa, then $D_{i}=D_{i+1}$. If the transformation of $\mathcal{P}_{i}$ to $\mathcal{P}_{i+1}$ is 


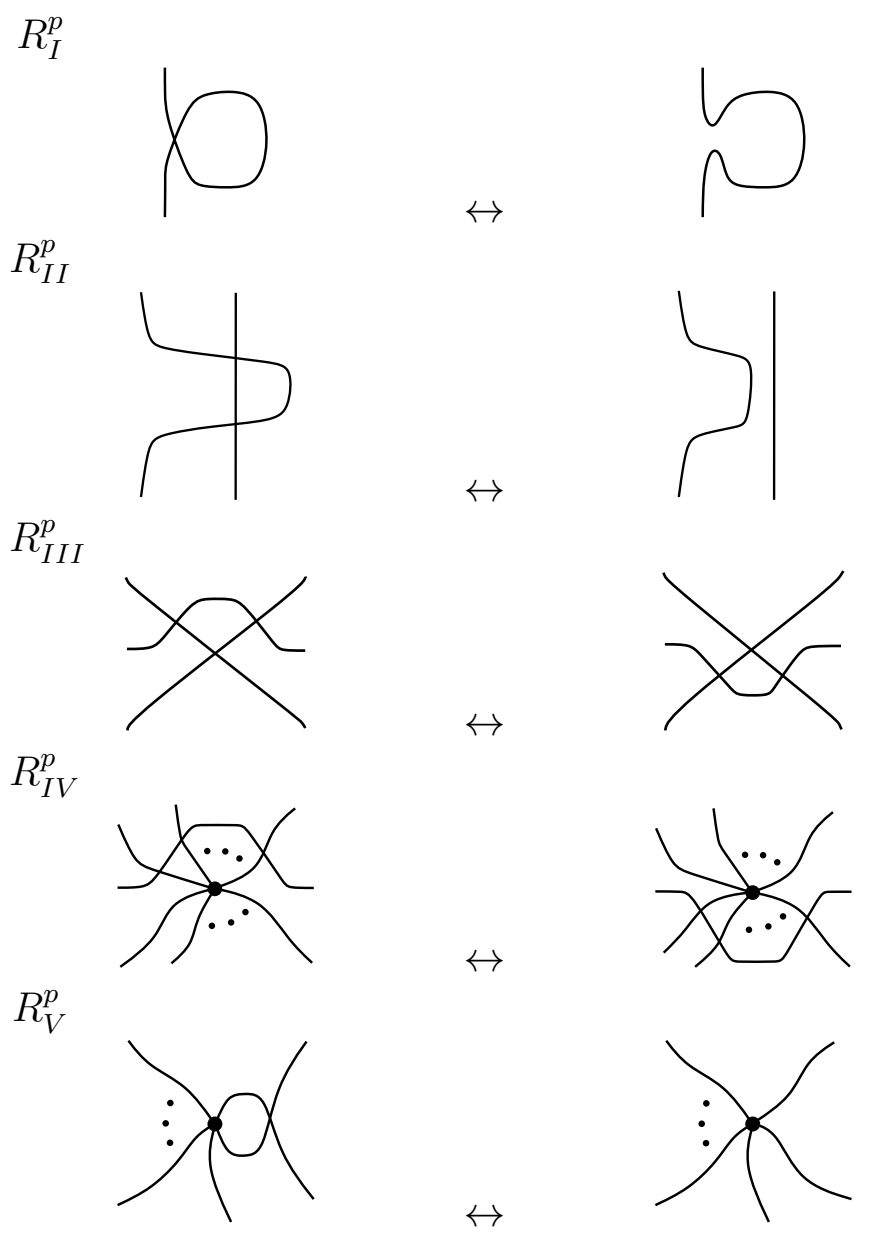

Table 2: Reidemeister moves for plane graphs where $R_{I V}^{p}$ and $R_{V}^{p}$ are shown for a vertex of degree 5 .

obtained by a Reidemeister move from Table 1 , then the transformation of $D_{i}$ to $D_{i+1}$ is obtained by the corresponding Reidemeister move from Table 2.

\section{Hanani-Tutte and Non-separating Planar Graphs}

A two-vertex-avoiding cycle in a graph $G$ is a cycle in $G$ with $|V(G)-2|$ vertices.

In any drawing $D$, define $\phi(D)$ to be the number of separating cycles in $D$ and define $\chi(D)$ to be the number of pairs of vertex-disjoint edges that cross each other an odd number of times in $D$.

For an edge $e$ and a vertex $v$ in a graph $G=(V, E)$, we denote:

- the set of all the two-vertex-avoiding cycles that have $e$ as an edge but do not have $v$ as a vertex by $C(e-v)$ and 
- the set of all the edges that are incident with $v$ but are vertex-disjoint from $e$ by $E(v-e)$.

Next we prove that the parity of $\phi+\chi$ is an invariant for drawings of some graphs that will be useful later.

Lemma 15. Let $G$ be a graph such that the shortest cycle in $G$ is a two-vertex-avoiding cycle and for any edge $e$ and any vertex $v$ that is not an endpoint of $e,|E(v-e)|$ and $|C(e-v)|$ have the same parity. Let $D$ and $D^{\prime}$ be two different drawings of $G$, then $\phi(D)+\chi(D)$ and $\phi\left(D^{\prime}\right)+\chi\left(D^{\prime}\right)$ have the same parity.

Proof. By Proposition 14, any drawing $D^{\prime}$ of $G$ can be obtained from $D$ by performing a series of Reidemeister moves in $\left\{R_{I}^{p}, R_{I I}^{p}, R_{I I I}^{p}, R_{I V}^{p}, R_{V}^{p}\right\}$. Therefore, in order to show that $\phi(D)+\chi(D)$ and $\phi\left(D^{\prime}\right)+\chi\left(D^{\prime}\right)$ have the same parity, it is enough to show that $\phi\left(D_{1}\right)+\chi\left(D_{1}\right)$ and $\phi\left(D_{2}\right)+\chi\left(D_{2}\right)$ have the same parity for any two drawings $D_{1}$ and $D_{2}$ of $G$ where $D_{2}$ is obtained by performing a Reidemeister move in $\left\{R_{I}^{p}, R_{I I}^{p}, R_{I I I}^{p}, R_{I V}^{p}, R_{V}^{p}\right\}$ on $D_{1}$.

Let $D_{2}$ be obtained from $D_{1}$ by performing any of the Reidemeister moves $R_{I}^{p}, R_{I I}^{p}$, $R_{I I I}^{p}, R_{V}^{p}$. In this case it is straightforward to see that $\phi\left(D_{1}\right)=\phi\left(D_{2}\right)$ and $\chi\left(D_{1}\right)=\chi\left(D_{2}\right)$ and therefore $\phi\left(D_{1}\right)+\chi\left(D_{1}\right)$ and $\phi\left(D_{1}\right)+\chi\left(D_{1}\right)$ have the same parity. So let $D_{2}$ be obtained from $D_{1}$ by performing a $R_{I V}^{p}$ move, in which edge $e$ is pushed over vertex $v$. We have two cases:

Case 1. Edge $\boldsymbol{e}$ is incident to $\boldsymbol{v}$. In this case, pushing $e$ over $v$ does not change the number of pairs of vertex-disjoint edges that cross each other and therefore $\phi\left(D_{1}\right)=\phi\left(D_{2}\right)$. Moreover, it does not change any separating cycle to a non-separating cycle or vice-versa and therefore $\chi\left(D_{1}\right)=\chi\left(D_{2}\right)$. Hence $\phi\left(D_{1}\right)+\chi\left(D_{1}\right)$ and $\phi\left(D_{2}\right)+\chi\left(D_{2}\right)$ have the same parity.

Case 2. Edge $\boldsymbol{e}$ is not incident to $\boldsymbol{v}$. Let $C_{s \rightarrow n}$ be the set of cycles in $G$ that are separating cycles in $D_{1}$ and are non-separating cycles in $D_{2}$ and let $C_{n \rightarrow s}$ be the set of cycles in $G$ that are non-separating cycles in $D_{1}$ and are separating cycles in $D_{2}$. Note that, as $e$ is pushed over $v$ :

- any cycle in $C(e-v)$ is a two-vertex-avoiding cycle and hence it either changes from a separating cycle to a non-separating cycle or vice-versa and

- any cycle that is not in $C(e-v)$ is either a cycle that contains all vertices of $G$ except for one of them or does not contain $e$ and hence remains a separating cycle or a non-separating cycle.

Therefore we have $\left|C_{s \rightarrow n}\right|+\left|C_{n \rightarrow s}\right|=|C(e-v)|$.

Now let $E_{o \rightarrow e}$ be the set of all edges in $G$ that cross $e$ an odd number of times in $D_{1}$ and an even number of times in $D_{2}$ and let $E_{e \rightarrow o}$ be the set of all edges in $G$ that cross $e$ an even number of times in $D_{1}$ and an odd number of times in $D_{2}$. As $e$ is pushed over $v$ :

- the number of crossings between $e$ and any edge $e^{\prime}$ which is in $E(v-e$ ) (and is therefore vertex-disjoint from $e$ ) either increases or decreases by one (so the parity of the number of crossings between $e$ and $e^{\prime}$ changes) and 
- the number of crossings between any other two vertex-disjoint edges remains the same.

Therefore we have $\left|E_{o \rightarrow e}\right|+\left|E_{e \rightarrow o}\right|=|E(v-e)|$.

Since $\left|C_{s \rightarrow n}\right|+\left|C_{n \rightarrow s}\right|$ has the same parity as $|C(e-v)|$ and $\phi\left(D_{2}\right)=\phi\left(D_{1}\right)-\left|C_{s \rightarrow n}\right|+$ $\left|C_{n \rightarrow s}\right|$, we find that $\phi\left(D_{1}\right)+\phi\left(D_{2}\right)$ has the same parity as $|C(e-v)|$. Moreover, since $\left|E_{o \rightarrow e}\right|+\left|E_{e \rightarrow o}\right|$ has the same parity as $|E(v-e)|$ and $\chi\left(D_{2}\right)=\chi\left(D_{1}\right)-\left|E_{o \rightarrow e}\right|+\left|E_{e \rightarrow o}\right|$, we find that $\chi\left(D_{1}\right)+\chi\left(D_{2}\right)$ has the same parity as $|E(v-e)|$. Therefore $\phi\left(D_{1}\right)+\phi\left(D_{2}\right)+$ $\chi\left(D_{1}\right)+\chi\left(D_{2}\right)$ has the same parity as $|C(e-v)|+|E(v-e)|$.

By the Lemma's assumption, the parities of $|E(v-e)|$ and $|C(e-v)|$ are the same. Therefore the parities of $\phi\left(D_{1}\right)+\chi\left(D_{1}\right)$ and $\phi\left(D_{2}\right)+\chi\left(D_{2}\right)$ are the same.

Now we can prove the following lemma.

Lemma 16. Any drawing of $K_{1} \cup K_{2,3}$ or $K_{1} \cup K_{4}$ or $K_{1,1,3}$ on the plane either contains a separating cycle or two vertex-disjoint edges that cross each other an odd number of times.

Proof. To prove this lemma we show that $\phi(D)+\chi(D)$ is odd in any drawing of $K_{1} \cup K_{2,3}$ or $K_{1} \cup K_{4}$ or $K_{1,1,3}$.

Let $G$ be $K_{1} \cup K_{2,3}$ or $K_{1} \cup K_{4}$ or $K_{1,1,3}$. It is routine to verify that a shortest cycle in $G$ is a two-vertex-avoiding cycle. Moreover it is routine to verify that for any edge $e$ in $G$ and any vertex $v$ in $G$ that is not an endpoint of $e,|C(e-v)|$ and $|E(v-e)|$ have the same parity. Therefore we can apply Lemma 15 to $G$.

Now let $D$ be a planar drawing of $G$. It is easy to see that $\phi(D)=1$ and $\chi(D)=0$ and therefore $\phi(D)+\chi(D)$ is odd. Moreover by Lemma 15, for any two drawings $D$ and $D^{\prime}$ of $G, \phi(D)+\chi(D)$ and $\phi\left(D^{\prime}\right)+\chi\left(D^{\prime}\right)$ have the same parity and hence $\phi+\chi$ is odd for any drawing of $G$.

We use this lemma to prove Theorem 1.

Proof of Theorem 1. By Lemma 16, we know that any drawing of $K_{1} \cup K_{4}, K_{1} \cup K_{2,3}$ or $K_{1,1,3}$ contains either a separating cycle or two vertex-disjoint edges that cross each other an odd number of times. It is easy to see that any drawing of a subdivision of $K_{1} \cup K_{4}$, $K_{1} \cup K_{2,3}$ or $K_{1,1,3}$ (or of a graph that contains any of $K_{1} \cup K_{4}, K_{1} \cup K_{2,3}$ or $K_{1,1,3}$ as a minor) also contains a separating cycle or two vertex-disjoint edges that cross each other an odd number of times. In other words, any non-separating drawing $D$ in which any two edges cross an even number of times does not contain a subdivision of $K_{1} \cup K_{4}, K_{1} \cup K_{2,3}$ or $K_{1,1,3}$ and therefore, by Theorem 11, it is a non-separating planar graph.

\section{Hanani-Tutte Theorem, Stronger Version}

Lemma 17. Let $D$ be a drawing of a graph $G$ on the plane which contains a separating cycle. Then there exists a chordless separating cycle in $D$. 
Proof. Let $C=\left(v_{1}, v_{2}, \ldots, v_{k}\right)$ be the shortest separating cycle in $D$. We claim that $C$ is chordless.

To the contrary, let $\left(v_{i}, v_{j}\right), 1 \leqslant i<j \leqslant k$, be a chord in $C$. Then we show that either cycle $C_{1}=\left(v_{1}, v_{2}, \ldots, v_{i}, v_{j}, v_{j+1}, \ldots, v_{k}\right)$ or cycle $C_{2}=\left(v_{i}, v_{i+1}, \ldots, v_{j}\right)$ is a shorter separating cycle than $C$ and hence we reach a contradiction.

Let $a$ and $b$ be two vertices that are separated by $C$ and let $\zeta$ be a curve from $a$ to $b$ that does not pass through any vertices or crossings of $D$ and is not tangent to any edge in $D$ at any point. Since $C$ is a separating cycle with respect to $a$ and $b$, the curve $\zeta$ crosses $C$ an odd number of times.

Now suppose that neither $C_{1}$ nor $C_{2}$ is separating. In other words, $\zeta$ crosses $C_{1}$ an even number of times and $\zeta$ crosses $C_{2}$ an even number of times.

Let $\alpha$ be the number of crossings between $\zeta$ and $C_{1}$, let $\beta$ be the number of crossings between $\zeta$ and $C_{2}$, and let $\gamma$ be the number of crossings between $\zeta$ and $\left(v_{i}, v_{j}\right)$. Then the number of crossings between $\zeta$ and $C$ is $\alpha+\beta-2 \gamma$ which is even, since $\alpha$ and $\beta$ are even, and therefore $C$ is not a separating cycle, which is a contradiction.

Now we are ready to prove Theorem 2 .

Proof of Theorem 2. We prove this theorem by induction on the number of chordless decomposing cycles in the drawing of the graph.

In the base case, there are no chordless decomposing cycles in $D$. By the definition of evenly decomposable drawings, any two vertex-disjoint edges in $D$ cross an even number of times.

First we show that $D$ is a non-separating drawing. To reach a contradiction suppose that there is a separating cycle in $D$. By Lemma 17, there exists a chordless separating cycle $C$ in $D$. Since any two vertex-disjoint edges in $D$ cross an even number of times, $C$ is a chordless decomposing cycle. This is a contradiction since according to the assumptions of the base case there are no chordless decomposing cycles in $D$.

Therefore $D$ is a non-separating drawing. By Theorem $1, G$ is a non-separating planar graph, so it has a planar drawing in which there are no separating cycles. Therefore the requirements of the conclusion of this theorem are satisfied.

We proceed to the inductive case where there is a chordless decomposing cycle $C=$ $\left(v_{1}, v_{2}, \ldots, v_{k}\right)$ in $D$. Cycle $C$ decomposes $G$ into two graphs $G_{w}=G\left[\left\{v_{1}, v_{2}, \ldots, v_{k}\right\} \cup V_{w}\right]$ and $G_{b}=G\left[\left\{v_{1}, v_{2}, \ldots, v_{k}\right\} \cup V_{b}\right]$ such that there is no edge between any vertex $v \in V_{w}$ of $G_{w}$ and any vertex $v^{\prime} \in V_{b}$ of $G_{b}$ (otherwise there is an edge in $D$ that crosses $C$ an odd number of times). In other words, the only vertices and edges that are shared between $G_{w}$ and $G_{b}$ are the vertices and the edges of $C$.

Since $D\left[G_{w}\right]$ is contained entirely within $D$, any cycle that is not a separating cycle in $D$ is not a separating cycle in $D\left[G_{w}\right]$ either. Moreover, since vertices of $V_{b}$ are not present in $D\left[G_{w}\right]$, the cycle $C$ - which was a separating cycle in $D$ - is not a separating cycle in $D\left[G_{w}\right]$. Therefore, the number of separating cycles in $D\left[G_{w}\right]$ is less than the number of separating cycles in $D[G]$. Similarly, the number of separating cycles in $D\left[G_{b}\right]$ is less than the number of separating cycles in $D[G]$. Hence by induction it follows that there is a planar drawing $D_{w}$ of $G_{w}$ such that all the vertices of $V_{w}$ are on the same side of $C$ and 
there is a planar drawing $D_{b}$ of $G_{b}$ such that all the vertices of $V_{b}$ are on the same side of $C$.

Since $C$ is not a separating cycle in $D_{w}$ and it does not have any chords, it is a face of $D_{w}$. Similarly, $C$ is a face of $D_{b}$. Therefore it is easy to see that we can obtain a drawing $D$ of $G$ by gluing $D_{w}$ and $D_{b}$ on the vertices and edges of $C$.

Figure 7 depicts an evenly decomposable drawing and its decomposition process. The drawing is decomposed a couple of times until there are no more decomposing cycles in it and any two vertex-disjoint edges cross each other an even number of times. Notice that in the first step of the decomposition we could have chosen the other cycle in the graph as the decomposing cycle.
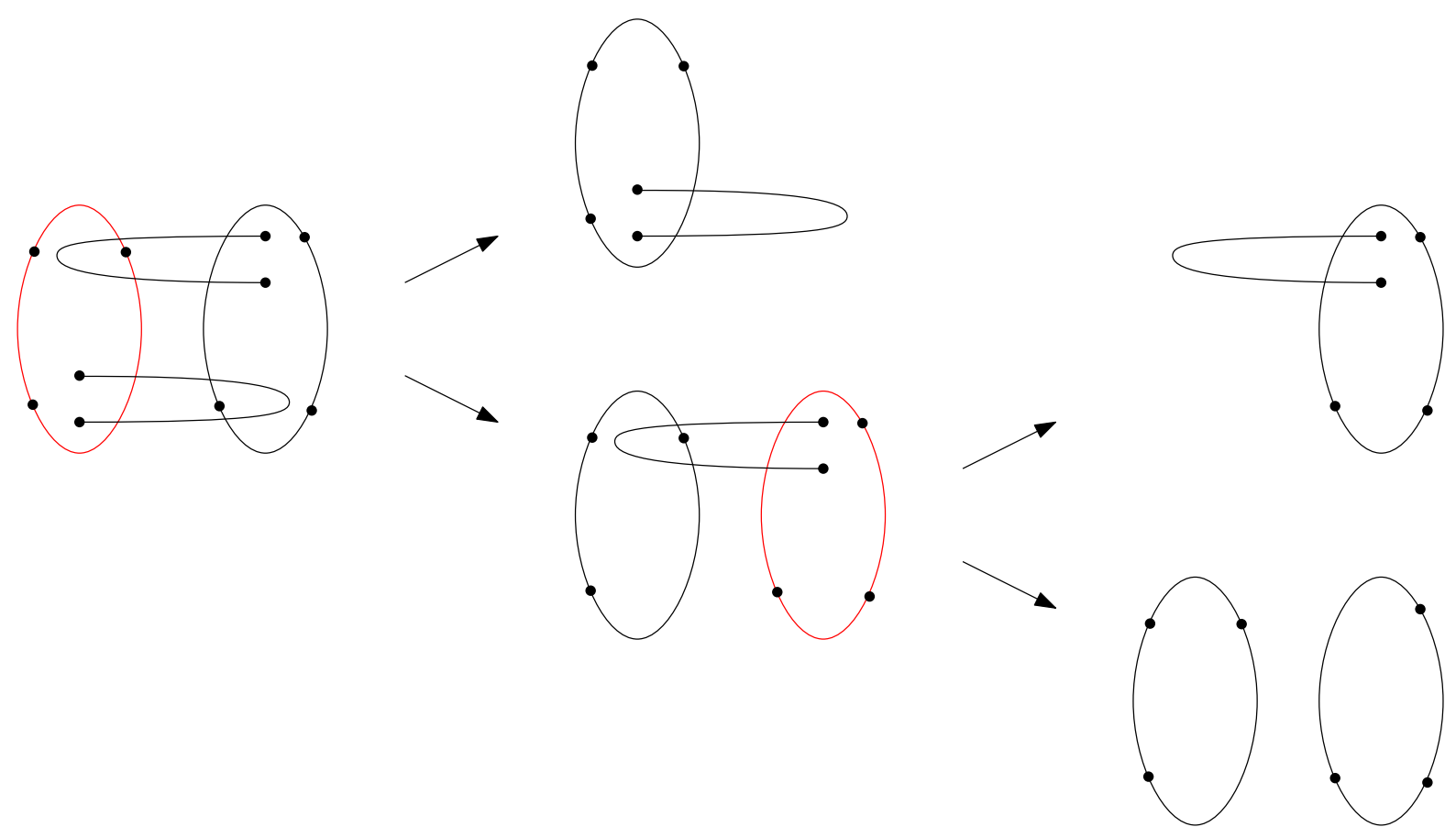

Figure 7: An evenly decomposable drawings and its decomposition. The decomposing cycle is depicted in red in each step. Notice that some of the edges of the decomposing cycles are crossed an odd number of times by other vertex-disjoint edges and hence the strong Hanani-Tutte Theorem is not applicable.

Since Theorem 2 is stronger than the strong version of the Hanani-Tutte Theorem, we can state the strong Hanani-Tutte Theorem as a corollary.

Corollary 18. (Strong Hanani-Tutte Theorem) Let D be a drawing of a graph $G$ such that any two vertex-disjoint edges in $D$ cross an even number of times. Then $G$ is planar.

Lastly, note that, since any planar drawing is an evenly decomposable drawing, Theorem 2 can also be stated as a characterisation of planar graphs.

Theorem 19. A graph $G$ is planar if and only if $G$ has an evenly decomposable drawing. 


\section{Conclusion}

In this paper we studied non-separating planar graphs. We used the forbidden minor characterisation of non-separating planar graphs to gave a Hanani-Tutte type characterisation for them in Theorem 1. It would also be worth proving Theorem 1 directly (without using the forbidden minor characterisation of non-separating planar graphs) as it could open up further opportunities for generalising Theorem 1.

Furthermore, we used non-separating planar graphs as a tool to decompose planar graphs. We believe the class of non-separating planar graphs deserves more attention in that they can provide us with better insights about other related classes of graphs such as planar graphs and linkless graphs (see for example [4]).

We then used the Hanani-Tutte type characterisation of non-separating planar graphs to prove Theorem 2 which is a stronger version of the strong Hanani-Tutte Theorem for planar graphs. Theorem 2 relies on the definition of evenly decomposable drawings and this definition is based on the requirements of Theorem 1. So Theorem 2 could be strengthened if one could provide a stronger Hanani-Tutte type characterisation for non-separating planar graphs.

Lastly, it would be interesting to know more about the class of graphs that are discussed in the statement of Lemma 15 and to know whether there are non-trivial graphs other than $K_{1} \cup K_{4}, K_{1} \cup K_{2,3}$ and $K_{1,1,3}$ that are included in this class of graphs.

\section{Acknowledgment}

The authors thank Peter Eades and David Wood for their helpful discussions and suggestions.

\section{References}

[1] C. C. Adams. The knot book: an elementary introduction to the mathematical theory of knots. American Mathematical Soc., 2004.

[2] G. Cairns and Y. Nikolayevsky. Bounds for generalized thrackles. Discrete \& Computational Geometry, 23(2):191-206, 2000.

[3] C. Chojnacki. Über wesentlich unplättbare Kurven im dreidimensionalen Raume. Fundamenta Mathematicae, 23(1):135-142, 1934.

[4] H. R. Dehkordi and G. Farr. Non-separating planar graphs. Electronic Journal of Combinatorics, 28(1):\#P1.11, 2021.

[5] R. Fulek and J. Kynčl. Counterexample to an extension of the Hanani-Tutte Theorem on the surface of genus 4. Combinatorica 39:1267-1279, 2019.

[6] R. Fulek, J. Kynčl, and D. Pálvölgyi. Unified Hanani-Tutte theorem. Electronic Journal of Combinatorics, 24(3), \#P3.18, 2017. 
[7] L. H. Kauffman. Invariants of graphs in three-space. Transactions of the American Mathematical Society, 311(2):697-710, 1989.

[8] C. Kuratowski. Sur le problème des courbes gauches en topologie. Fundamenta Mathematicae, 15(1):271-283, 1930.

[9] M. Loebl and G. Masbaum. On the optimality of the Arf invariant formula for graph polynomials. Advances in Mathematics, 226(1):332 - 349, 2011.

[10] M. J. Pelsmajer, M. Schaefer, and D. Stasi. Strong Hanani-Tutte on the projective plane. SIAM Journal on Discrete Mathematics, 23(3):1317-1323, 2009.

[11] M. J. Pelsmajer, M. Schaefer, and D. Štefankovič. Removing even crossings on surfaces. European Journal of Combinatorics, 30(7):1704-1717, 2009.

[12] W. T. Tutte. Toward a theory of crossing numbers. Journal of Combinatorial Theory, 8(1):45-53, 1970.

[13] S. Yamada. An invariant of spatial graphs. Journal of Graph Theory, 13(5):537-551, 1989. 